\title{
Modulation of bone morphogenetic protein signaling in vivo regulates systemic iron balance
}

\author{
Jodie L. Babitt, ${ }^{1}$ Franklin W. Huang, ${ }^{2}$ Yin Xia, ${ }^{1}$ Yisrael Sidis, ${ }^{1}$ Nancy C. Andrews, ${ }^{2}$ and Herbert Y. Lin ${ }^{1}$ \\ 1Program in Membrane Biology and Nephrology Division, Massachusetts General Hospital, Harvard Medical School, Boston, Massachusetts, USA. \\ ${ }^{2}$ Children's Hospital Boston and Dana-Farber Cancer Institute, Harvard Medical School, Boston, Massachusetts, USA.
}

\begin{abstract}
Systemic iron balance is regulated by hepcidin, a peptide hormone secreted by the liver. By decreasing cell surface expression of the iron exporter ferroportin, hepcidin decreases iron absorption from the intestine and iron release from reticuloendothelial stores. Hepcidin excess has been implicated in the pathogenesis of anemia of chronic disease, while hepcidin deficiency has a key role in the pathogenesis of the iron overload disorder hemochromatosis. We have recently shown that hemojuvelin is a coreceptor for bone morphogenetic protein (BMP) signaling and that BMP signaling positively regulates hepcidin expression in liver cells in vitro. Here we show that BMP-2 administration increases hepcidin expression and decreases serum iron levels in vivo. We also show that soluble hemojuvelin (HJV.Fc) selectively inhibits BMP induction of hepcidin expression in vitro and that administration of HJV.Fc decreases hepcidin expression, increases ferroportin expression, mobilizes splenic iron stores, and increases serum iron levels in vivo. These data support a role for modulators of the BMP signaling pathway in treating diseases of iron overload and anemia of chronic disease.
\end{abstract}

\section{Introduction}

Anemia of chronic disease, also known as anemia of inflammation, is prevalent in patients with many systemic diseases including autoimmune disorders, malignancy, and chronic kidney disease (1). In these patients intestinal iron absorption is impaired and iron remains sequestered in reticuloendothelial cells, leading to hypoferremia and anemia (1). Research over the last several years implicates hepcidin excess in the pathogenesis of this disease (1-6). A key regulator of systemic iron homeostasis (7), hepcidin is secreted by the liver $(2,8,9)$ and induces internalization and degradation of the iron exporter ferroportin in absorptive enterocytes and reticuloendothelial cells, thereby inhibiting iron absorption from the intestine and iron release from reticuloendothelial cell stores (10). Hepcidin expression is inhibited by anemia and hypoxia, thus increasing iron availability when needed for erythropoiesis (3). Conversely, hepcidin expression is induced by iron loading, thus providing a feedback mechanism to limit further iron absorption (2-4). Hepcidin expression is also induced by inflammatory cytokines, and this is thought to be the mechanism underlying the impaired intestinal iron absorption, reticuloendothelial cell iron sequestration, and hypoferremia characteristic of anemia of chronic disease (1-6).

While hepcidin excess has a role in anemia of chronic disease, inadequate hepcidin expression appears to be a common pathogenic mechanism for the iron overload disorder hereditary hemochromatosis as a result of mutations in the genes encoding hepcidin (HAMP), hemojuvelin (HFE2), HFE, or transferrin receptor 2 (TFR2) (11-21). In patients and animal models with iron overload due to mutations in these genes, hepcidin levels are low, thereby leading to ferroportin

Nonstandard abbreviations used: BMP, bone morphogenetic protein; GDF, growth and differentiation factor; HJV.Fc, soluble hemojuvelin comprised of the extracellular domain of hemojuvelin fused to the Fc portion of human IgG; pRL-TK, Renilla luciferase vector.

Conflict of interest: H.Y. Lin's laboratory received research funding from GlaxoSmithKline.

Citation for this article: J. Clin. Invest. 117:1933-1939 (2007). doi:10.1172/JCI31342. overactivity, increased intestinal iron absorption, increased reticuloendothelial cell iron release, elevated serum iron levels, and abnormal tissue iron deposition (11-21). Although the mechanisms by which mutations in HFE and TFR2 lead to low hepcidin levels remain unclear, emerging evidence suggests that hemojuvelin functions as a coreceptor for bone morphogenetic protein (BMP) signaling (22) and that BMP/TGF- $\beta$ superfamily signaling has a role in regulating hepcidin expression and systemic iron balance (22-24).

Members of the BMP/TGF- $\beta$ superfamily, including BMPs, TGF- $\beta$ s, growth and differentiation factors (GDFs), and activins, initiate an intracellular signaling cascade by binding to a complex of type I and type II serine threonine kinase receptors (25). The activated receptor complex phosphorylates intracellular Smad proteins, which then complex with common mediator Smad4. Smad complexes translocate to the nucleus where they modulate gene transcription. In general, BMPs and GDFs signal via one set of Smad proteins (Smad1, Smad5, and Smad8), while TGF- $\beta$ s and activins signal via another set (Smad2 and Smad3).

Hemojuvelin (also known as RGMc) is a member of the repulsive guidance molecule (RGM) family, which includes RGMa and DRAGON (also known as RGMb) $(13,26,27)$. We have recently demonstrated that, similar to RGMa and DRAGON $(28,29)$, hemojuvelin functions as a BMP coreceptor that binds directly to BMP-2 and BMP-4 and enhances cellular responses to BMP, but not TGF- $\beta$, ligands (22). Furthermore, BMP-2 positively regulates hepcidin expression $(22,24)$, and hemojuvelin increases hepcidin induction in response to BMP-2 (22). Hemojuvelin mutants associated with juvenile hemochromatosis have impaired BMP signaling ability, and hepatocytes from $\mathrm{H} f e 2^{-/-}$mice demonstrate blunted hepcidin induction in response to BMP-2 (22). This suggests that the mechanism for iron overload in patients with hemojuvelin mutations is a result of decreased BMP signaling in the liver leading to decreased hepcidin expression.

Further evidence supporting a role for BMP signaling in regulating hepcidin expression and iron metabolism in vivo comes from 

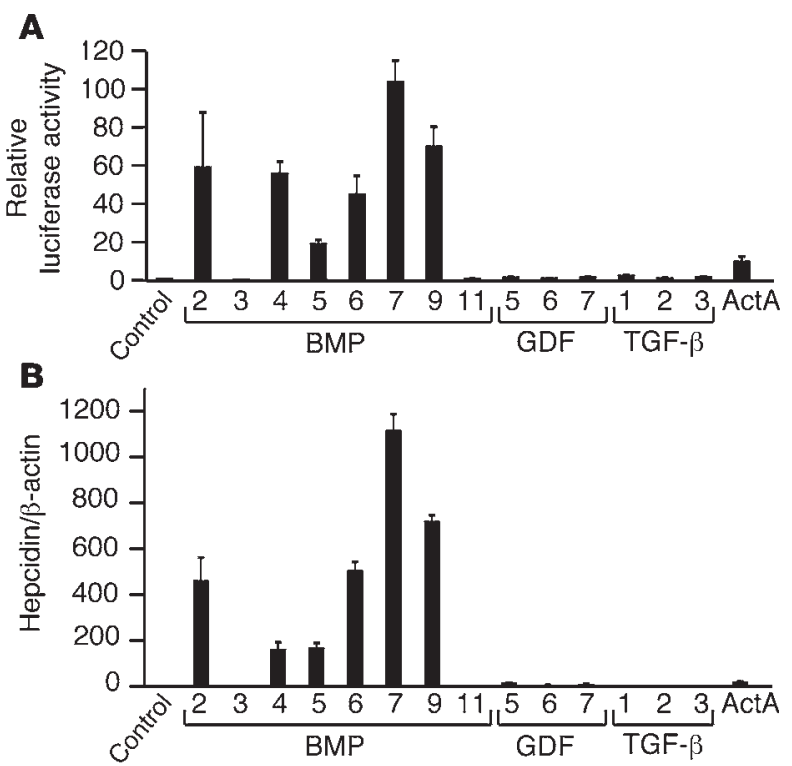

mice with a conditional liver-specific knockout of Smad4. These mice have low hepcidin levels and develop iron overload (23). In that study, both BMP-4 and TGF- $\beta 1$ were shown to induce hepcidin expression in liver cells in vitro (23). Hepcidin induction by BMP-9 has also been described (24).

Here we test a wide array of TGF- $\beta$ superfamily members to compare their relative abilities to regulate hepcidin expression in vitro. We also investigate the ability of BMP-2 to positively regulate hepcidin expression and reduce serum iron levels in vivo. Finally, we generate soluble hemojuvelin, comprised of the extracellular domain of hemojuvelin fused to the Fc portion of human IgG (HJV.Fc) and examine the ability of soluble hemojuvelin to inhibit hepatic BMP signaling, decrease hepcidin expression, increase ferroportin expression, mobilize reticuloendothelial iron stores, and increase serum iron levels.

\section{Results}

Selective regulation of hepcidin by BMP/TGF- $\beta$ superfamily members. TGF- $\beta$ superfamily members were tested for their ability to regulate hepcidin using both a hepcidin promoter reporter assay (Figure 1A) and quantitative real-time RT-PCR (Figure 1B) in Hep3B hepatoma-derived cells. Relative concentrations of BMP/TGF- $\beta$ superfamily ligands used are similar to those previously used by others to compare responses among superfamily ligands $(23,30,31)$. BMP-2, BMP-4, BMP-5, BMP-6, BMP-7, and BMP-9 robustly increased hepcidin promoter luciferase activity 20- to 100-fold over baseline and increased hepcidin mRNA expression by 160 - to 1,100-fold. In contrast, TGF- $\beta 1,-\beta 2$, and $-\beta 3$ increased hepcidin expression by only 1.5 - to 3 -fold over baseline by both methods. BMP-3, BMP-11, GDF-5, GDF-6, and GDF-7 showed no or comparatively little hepcidin induction by both methods. Activin A increased hepcidin promoter relative luciferase activity by 10 -fold but increased hepcidin mRNA expression to a lesser extent relative to BMP-2, BMP-4, BMP-5, BMP-6, BMP-7, and BMP-9 as analyzed by real-time RT-PCR. Biologic activity of all ligands was verified by luciferase assay using a BMP-responsive firefly luciferase reporter (30) and a TGF- $\beta$ /activinresponsive firefly luciferase reporter (31). Results using both methods correlated well with each other, suggesting that the hepcidin

\section{Figure 1}

Induction of hepcidin expression by TGF- $\beta$ /BMP superfamily ligands. (A) Hep3B cells were transfected with a hepcidin promoter firefly luciferase reporter and a control pRL-TK. Transfected cells were incubated either alone (control) or with $50 \mathrm{ng} / \mathrm{ml} \mathrm{BMP}$ or GDF ligands, $5 \mathrm{ng} / \mathrm{ml}$ TGF- $\beta$ ligands, or $30 \mathrm{ng} / \mathrm{ml}$ activin A (ActA) as indicated. Cell lysates were analyzed for luciferase activity. To control for transfection efficiency, relative luciferase activity was calculated as the ratio of firefly luciferase values to Renilla luciferase values and is expressed as the fold increase compared with control. Results are reported as the mean $\pm S D$ ( $n=2-3$ per group). (B) Hep3B cells were treated with BMP, GDF, TGF- $\beta$, or activin A ligands as in A. Total RNA was analyzed by quantitative real-time RT-PCR for hepcidin mRNA expression and $\beta$-actin mRNA expression. Samples were analyzed in triplicate and are reported as the ratio of the mean values of hepcidin to $\beta$-actin.

promoter luciferase assay is a good surrogate for hepcidin mRNA expression by quantitative real-time RT-PCR. Thus, many TGF- $\beta$ superfamily members can positively regulate hepcidin expression in vitro; however, BMP-2, BMP-4, BMP-5, BMP-6, BMP-7, and BMP-9 are much more potent regulators of hepcidin compared with other superfamily members, including all 3 TGF- $\beta$ ligands.

BMP-2 administration in vivo increases hepcidin expression and decreases serum iron. We next investigated whether BMP-2 regulates hepcidin expression and iron metabolism in vivo. Purified BMP-2 at $1 \mathrm{mg} / \mathrm{kg}$ was injected retroorbitally into mice, followed by determination of serum iron levels and hepatic hepcidin mRNA expression 4 hours after injection. BMP-2 administration increased hepatic hepcidin mRNA expression 1.8-fold over mice injected with vehicle alone (Figure 2A; $P=0.02$ ). BMP-2 administration also decreased serum iron levels from $170 \mu \mathrm{g} / \mathrm{dl}$ to $114 \mu \mathrm{g} / \mathrm{dl}$ (Figure 2B; $P=0.02$ ). This is consistent with a role for BMP-2 as a positive regulator of hepcidin expression in vivo.

Soluble HJV.Fc selectively inhibits BMP signaling in vitro. Soluble receptors such as the soluble TNF receptor etanercept have previously been used to inhibit ligand activity in vitro and in vivo, presumably by binding to ligands and preventing their interaction with membrane-bound receptors (32). Interestingly, soluble hemojuvelin has been detected in human sera and has been shown to inhibit hepcidin expression in cultured cells, although the mechanism for this inhibition was not investigated (33). We therefore generated purified soluble HJV.Fc (Figure 3A), the murine homolog of which
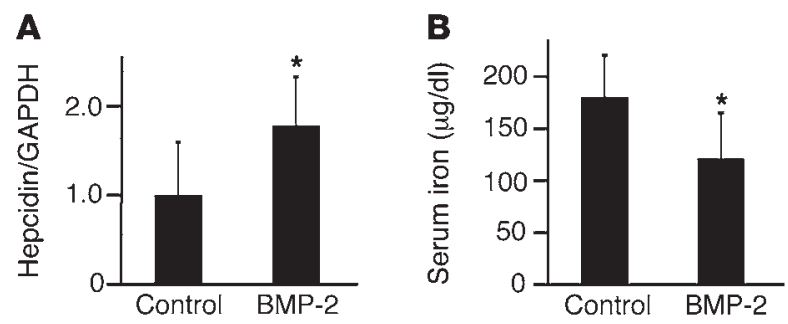

\section{Figure 2}

BMP-2 administration in mice increases hepcidin mRNA expression and decreases serum iron. 129S6/SvEvTac mice were injected retroorbitally with $1 \mathrm{mg} / \mathrm{kg} \mathrm{BMP}-2(n=8)$ or an equal volume of vehicle alone $(n=7)$. Four hours after injection, blood and livers were harvested. (A) Total mRNA was isolated from livers and analyzed by quantitative real-time RT-PCR for hepcidin mRNA expression relative to expression of GAPDH mRNA, which was used as an internal control. (B) Serum iron was measured by colorimetric assay. Results are reported as the mean \pm SD. ${ }^{\star} P=0.02$ for BMP-2-treated mice compared with controls. 
A
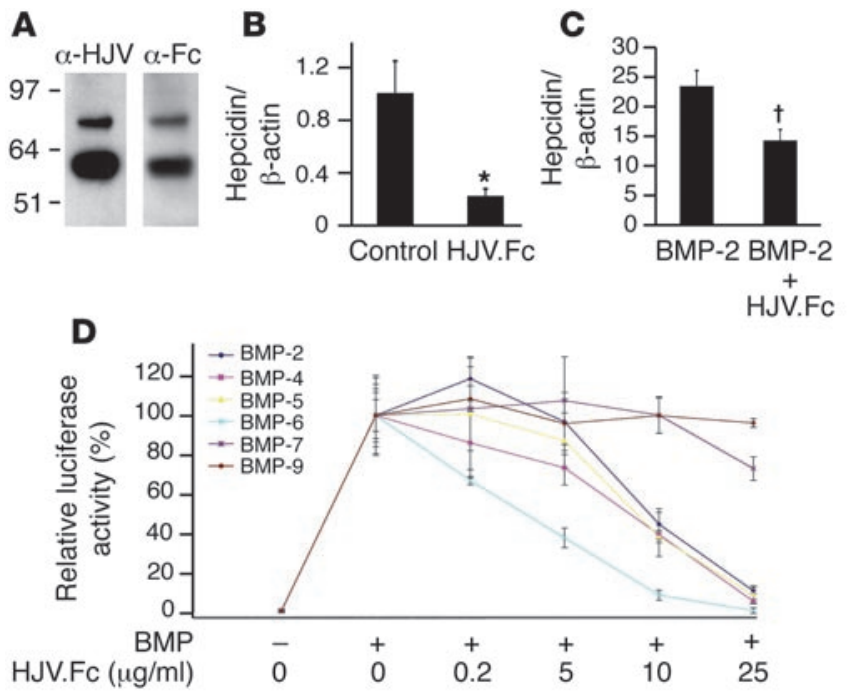

we have previously shown can bind to BMP-2 and BMP-4 ligands (22). We then investigated whether HJV.Fc inhibited basal hepcidin expression and BMP induction of hepcidin expression in vitro. In hepatoma-derived HepG2 cells, which have higher basal hepcidin expression, HJV.Fc inhibited basal hepcidin mRNA expression by $80 \%$ (Figure 3B; $P=0.03$ ). These results are consistent with a prior report using soluble hemojuvelin that does not contain an Fc tail (33), suggesting that the Fc tail does not affect the function of soluble hemojuvelin. HJV.Fc also inhibited BMP-2 induction of hepcidin expression (Figure 3C; $P=0.009$ ) and BMP-2-induced activation of the hepcidin promoter in a dose-dependent fashion (Figure 3D). HJV.Fc inhibition of BMP ligands was selective: HJV.Fc inhibited more than $90 \%$ of hepcidin promoter activation induced by BMP-2, BMP-4, BMP-5, and BMP-6 but did not inhibit BMP-9 even at lower ligand concentrations (Figure 3D). There was a trend toward low-level inhibition of BMP-7 (Figure 3D).

\section{Figure 3}

Soluble HJV.Fc inhibits basal hepcidin expression and selectively inhibits BMP induction of hepcidin expression. (A) Western blot of purified soluble HJV.Fc fusion protein with anti-hemojuvelin antibody $(\alpha-H J V)$ and anti-Fc antibody $(\alpha-F c)$. (B and $\mathbf{C})$ HepG2 cells were incubated alone (control) or with $25 \mu \mathrm{g} / \mathrm{ml} \mathrm{HJV.Fc} \mathrm{alone,} 25 \mathrm{ng} / \mathrm{ml} \mathrm{BMP-2}$ alone, or a combination of HJV.Fc and BMP-2 as indicated. Total RNA was isolated and quantitative real-time RT-PCR for hepcidin mRNA relative to $\beta$-actin mRNA was performed as in Figure 1. Results are reported as the mean $\pm \mathrm{SD}\left(n=3\right.$ per group; ${ }^{*} P=0.03$ for HJV.FC compared with control; $\uparrow P=0.009$ for HJV.Fc plus BMP-2 compared with BMP-2 alone). (D) Hep3B cells were transfected with the hepcidin promoter luciferase construct and $\mathrm{pRL}-\mathrm{TK}$. Transfected cells were incubated alone, with $5 \mathrm{ng} / \mathrm{ml} \mathrm{BMP}-9,50 \mathrm{ng} / \mathrm{ml} \mathrm{BMP}-5$, or $25 \mathrm{ng} / \mathrm{ml}$ BMP-2, BMP-4, BMP-6, or BMP-7 ligands, or with the BMP ligands plus 0.2 to $25 \mu \mathrm{g} / \mathrm{ml} \mathrm{HJV}$.Fc as indicated, followed by measurement of relative luciferase activity as in Figure 1. Results are reported as the mean \pm SD of the percent decrease in relative luciferase activity for cells treated with BMP ligands in combination with HJV.Fc compared with cells treated with BMP ligands alone ( $n=2$ per group).

We have previously demonstrated that BMP-2 and BMP-4 are endogenously expressed in HepG2 cells (22), and we hypothesized that inhibition of these endogenous BMP ligands was the mechanism by which HJV.Fc decreased basal hepcidin expression in HepG2 cells. We therefore used RT-PCR to investigate whether other BMP ligands are endogenously expressed in HepG2. We then tested whether siRNA inhibition of these endogenously expressed BMP ligands inhibited basal hepcidin expression in a manner similar to HJV.Fc. BMP-2, BMP-4, and BMP-6 were endogenously expressed in HepG2 cells, with BMP-4 being the most abundant (Figure 4A). BMP-2, BMP-4, and BMP-6 siRNA each selectively and significantly reduced endogenous ligand expression in HepG2 cells by 65\%, 90\%, and $55 \%$, respectively, as measured by real-time RT-PCR (Figure 4B). BMP-2, BMP-4, and BMP-6 siRNA each significantly inhibited basal hepcidin expression in HepG2 cells by approximately $10 \%$ $(P=0.012), 35 \%(P=0.0027)$, and $15 \%(P=0.0026)$, respectively, as
A

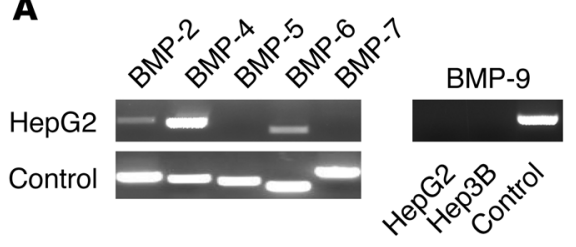

B

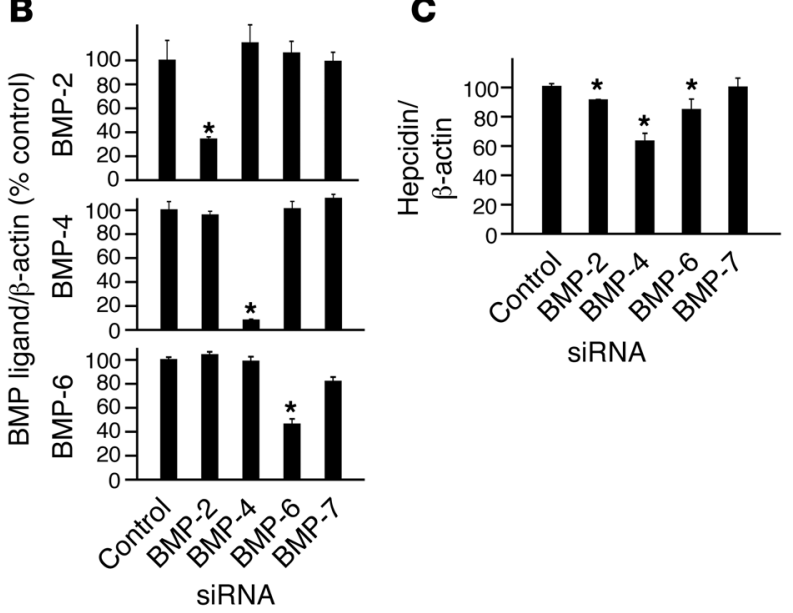

Figure 4

siRNA inhibition of endogenous BMP ligands decreases basal hepcidin expression. (A) Expression of endogenous BMP ligands in HepG2 cells as measured by RT-PCR. Purified plasmid cDNAs expressing BMP ligands were used as positive controls. (B and C) HepG2 cells were transfected with BMP ligand siRNAs or a control scrambled siRNA as indicated. Total RNA was analyzed for BMP ligand expression (B) or hepcidin expression (C) relative to $\beta$-actin expression by real-time quantitative RT-PCR. Results are reported as the mean \pm SD of the percent decrease in the ratio of hepcidin or BMP ligand to $\beta$-actin for cells treated with various BMP siRNAs compared with cells treated with control siRNA; $n=3-6$ per group; ${ }^{*} P<0.05$. 
A
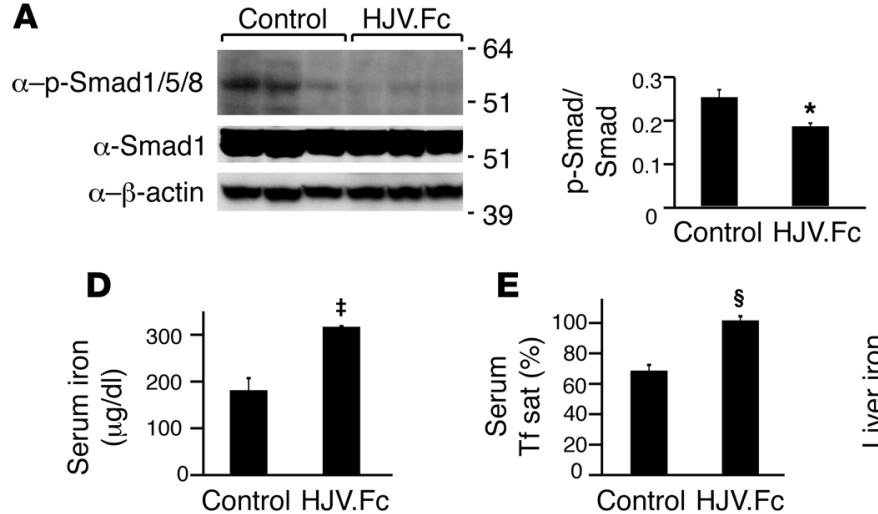

E

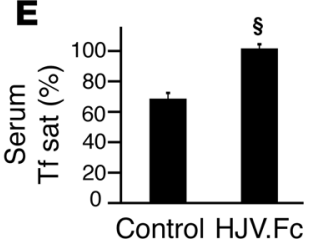

B
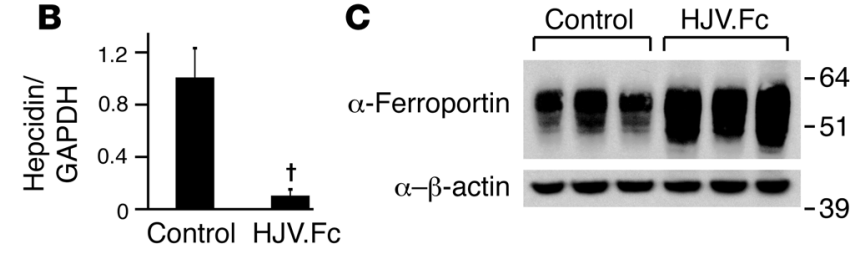

$\mathbf{F}$

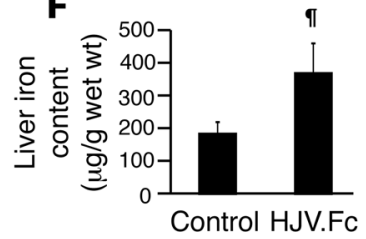

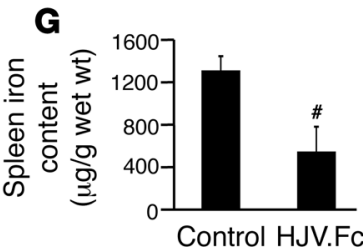

\section{Figure 5}

Soluble HJV.Fc administration in mice decreases hepatic phosphorylated Smad1, Smad5, and Smad8 expression, decreases hepcidin expression, increases serum iron, increases liver iron content, and decreases spleen iron content. 129S6/SvEvTac mice received an i.p. injection of $25 \mathrm{mg} / \mathrm{kg} \mathrm{HJV.Fc} \mathrm{or} \mathrm{normal} \mathrm{saline} \mathrm{(control)} 3$ times weekly for 3 weeks. (A) Liver lysates were analyzed for phosphorylated Smad1, Smad5, and Smad8 $(\alpha-p-S m a d 1 / 5 / 8)$ expression by western blot. Blots were stripped and reprobed for expression of total Smad1 and $\beta$-actin, which were used as loading controls. Chemiluminescence was quantitated by IPLab Spectrum software for phosphorylated Smad1, Smad5, and Smad8 expression relative to total Smad1 expression. (B) Total mRNA was isolated from livers and analyzed by quantitative real-time PCR for hepcidin mRNA expression relative to GAPDH mRNA expression as an internal control. (C) Spleen membrane preparations were analyzed for ferroportin expression by western blot. Blots were stripped and reprobed for expression of $\beta$-actin, which was used as a loading control. (D and E) Measurement of serum iron (D) and transferrin saturation (Serum Tf sat; E). ( $\mathbf{F}$ and $\mathbf{G}$ ) Quantitation of liver $(\mathbf{F})$ and spleen $(\mathbf{G})$ tissue iron content. Results are expressed as mean $\pm \mathrm{SD}, n=3$ mice per group; ${ }^{\star} P=0.0497,{ }^{\dagger} P=0.003,{ }^{\ddagger} P=0.01,{ }^{\S} P=0.004,{ }^{\natural} P=0.03,{ }^{\sharp} P=0.009$ for HJV.Fc-treated mice compared with controls.

measured by real-time RT-PCR (Figure 4C). As a negative control, neither a control siRNA nor a BMP-7-specific siRNA inhibited basal hepcidin expression. The relative ability of each ligand to inhibit basal hepcidin correlated with the relative mRNA abundance of the ligand and the strength of siRNA inhibition of ligand expression. These data suggest that endogenous BMP-2, BMP-4, and BMP-6 ligands all contribute to basal hepcidin expression in HepG2 cells. These data are consistent with our hypothesis that the mechanism by which HJV.Fc inhibits basal hepcidin expression in these cells is by inhibiting endogenous BMP signaling, presumably by binding and sequestering endogenously produced BMP ligands and preventing their interaction with BMP type I and type II receptors.

Soluble HJV.Fc inhibits hepatic BMP signaling, inhibits hepcidin expression, increases ferroportin expression, mobilizes reticuloendothelial cell iron stores, and increases serum iron in vivo. To test whether HJV.Fc administration could regulate hepcidin expression and iron metabolism in vivo, mice were injected with $25 \mathrm{mg} / \mathrm{kg}$ purified HJV.Fc or an equal volume of normal saline by i.p. injection 3 times weekly for 3 weeks. Western blot analysis of liver lysates from these mice showed decreased phosphorylated Smad1, Smad5, and Smad8 expression relative to total Smad1 expression in HJV.Fc-treated mice compared with control mice (Figure 5A; $P=0.0497$ ), demonstrating that HJV.Fc decreases hepatic BMP signaling in vivo. Quantitative real-time RT-PCR analysis revealed a 10-fold decrease in hepatic hepcidin mRNA expression in HJV.Fc-treated mice compared with control mice (Figure 5B; $P=0.003$ ). Consistent with the predicted effects of depressed hepcidin levels to increase ferroportin cell surface expression, increase intestinal iron absorption, and increase release of iron from reticuloendothelial stores, HJV.Fc treatment increased ferroportin expression in the spleen compared with control mice, as measured by western blot (Figure 5C). HJV.Fc treatment also increased serum iron levels from $177 \pm 26 \mu \mathrm{g} / \mathrm{dl}$ to $309 \pm 2 \mu \mathrm{g} / \mathrm{dl}$ (Figure 5D; $P=0.01$ ) and increased serum transferrin saturation from $70 \%$ to $100 \%$ (Figure 5E; $P=0.004$ ). Furthermore, HJV.Fc treatment increased hepatic tissue iron content by approximately 2 -fold (Figure 5F; $P=0.03$ ) and reduced splenic tissue iron content by almost 60\% (Figure 5G; $P=0.009$ ).

Soluble HJV.Fc inbibits IL-6 induction of hepcidin expression. Inflammatory cytokines induce hepcidin expression, and this hepcidin excess is thought to play a role in the anemia of chronic disease (1-6). We therefore investigated whether HJV.Fc could inhibit hepcidin induction by the inflammatory cytokine IL-6. IL-6 increased hepcidin expression 3.3-fold in HepG2 cells as measured by realtime RT-PCR (Figure 6; $P=0.003$ ). Hepcidin induction by IL-6 was significantly abrogated when cells were incubated with HJV.Fc in combination with IL-6 (Figure 6; $P=0.0006$ compared with cells treated with IL-6 alone).

\section{Discussion}

Hepcidin deficiency is the common pathogenic mechanism for both juvenile and adult forms of the genetic iron overload disorder hereditary hemochromatosis as a result of mutations in HAMP, HFE2, TFR2, and HFE (11-21). We have previously shown that hemojuvelin acts as a coreceptor for BMP signaling and that BMP-2 signaling induces hepcidin expression in vitro (22). Here we show that BMP-2 administration in mice increases hepcidin expression and reduces serum iron levels in vivo. These data support our in vitro data that BMP-2 can positively regulate hepcidin expression in vivo. The modest induction of hepcidin expression in response to BMP-2 in vivo compared with our in vitro findings is likely multifactorial. First, the mice were maintained on a standard diet, where dietary iron is replete, basal hepcidin levels are generally high, and hepcidin induction by well established regulators such as iron and LPS have been reported to be absent or less robust compared with mice maintained on an iron-deficient diet (4). Indeed, the degree of hepcidin induction by BMP- 2 in our study was similar to the 1.8 -fold induction reported 


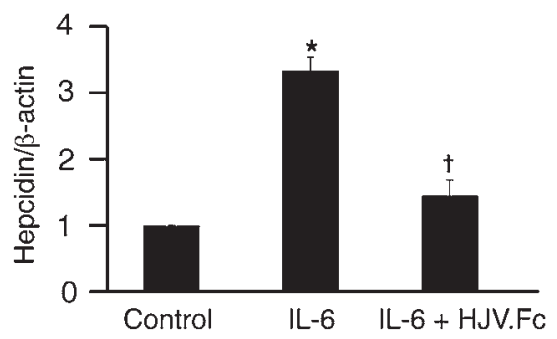

Figure 6

Soluble HJV.Fc inhibits IL-6 induction of hepcidin expression. HepG2 cells were incubated for 16 hours alone (control), with $100 \mathrm{ng} / \mathrm{ml} \mathrm{IL-6,}$ or with $100 \mathrm{ng} / \mathrm{ml} \mathrm{IL-6}$ in combination with HJV.Fc after pre-incubation with HJV.Fc for 1 hour. Total RNA was analyzed for hepcidin expression relative to $\beta$-actin expression by quantitative real-time RT-PCR. Results are expressed as mean $\pm \mathrm{SD}, n=3$ per group; ${ }^{*} P=0.003$ for IL-6-treated cells compared with control cells; ${ }^{+} P=0.0006$ for cells treated with HJV.Fc in combination with IL-6 compared with cells treated with IL-6 alone.

after LPS administration in mice fed on a standard diet (34). Furthermore, BMPs typically act in an autocrine or paracrine manner in vivo, while i.v. administered BMP-2 has been documented to be rapidly eliminated from the systemic circulation $\left(t_{1 / 2}=16 \mathrm{~min}\right)$ (35). Thus, it is likely that the systemically administered BMP-2 dose was not delivered efficiently to the liver. Nevertheless, the decrease in serum iron suggests that the BMP-2-induced increase in hepcidin expression was physiologically relevant and presumably reflects decreased iron export from reticuloendothelial cells and duodenal enterocytes due to hepcidin-induced internalization and degradation of ferroportin. Although treatment with systemic BMP-2 itself may be impractical due to its high cost and rapid elimination from the systemic circulation, our data suggest that therapies that enhance hepatic BMP signaling may provide alternative treatment strategies for managing iron overload in patients with hereditary hemochromatosis.

Liver-specific conditional Smad4 knockout mice have reduced hepcidin expression and total body iron overload, underscoring the important role for TGF- $\beta$ /BMP superfamily members in regulating hepcidin expression and iron metabolism in vivo (23). While our data demonstrate that BMP-2 can positively regulate hepcidin expression in vivo, it remains unknown which superfamily ligands are the endogenous regulators of hepcidin expression, since Smad4 is the common downstream mediator for all superfamily members. Indeed, many superfamily members are endogenously expressed in the liver, including BMP-2, BMP-4, BMP-5, BMP-6, BMP-9, and all 3 TGF- $\beta$ ligands (36-40 and J.L. Babitt, unpublished observations). Whether the liver is in fact the source of the endogenous BMP superfamily ligands, however, remains uncertain. Our previous data showing that hemojuvelin is a coreceptor for BMP, but not TGF- $\beta$, signaling (22) suggests that members of the BMP subfamily are more important than members of the TGF- $\beta$ subfamily for regulating iron metabolism in vivo. Here we show that many members of the TGF- $\beta$ superfamily can induce hepcidin mRNA expression in vitro. However, our results suggest that a subset of BMP ligands including BMP-2, BMP-4, BMP-5, BMP-6, BMP-7, and BMP-9 are much more potent inducers of hepcidin expression than other ligands tested, including all 3 TGF- $\beta$ ligands. Our results showing 3 -fold induction of hepcidin expression by TGF- $\beta 1$ are consistent with prior findings (23); however, we found that BMP-4 and BMP-9 were much more potent inducers of hepcidin expression compared with prior studies (23-24). This may be in part related to differences in ligand concentration (2- to 5-fold higher in our study) and/or differences in cell lines used. Interestingly, although BMP-9 is expressed in the liver (40) and can robustly increase hepcidin mRNA expression in vitro, HJV.Fc was unable to inhibit BMP-9 activation of the hepcidin promoter. HJV.Fc also had reduced ability to inhibit BMP-7 compared with BMP-2, BMP-4, BMP-5, and BMP-6 ligands, consistent with our prior findings (22). The ability of HJV.Fc to inhibit hepcidin expression and increase serum iron in vivo therefore suggests that BMP-2, BMP-4, BMP-5, and/or BMP-6 are good candidates for endogenous regulators of hepcidin expression, while BMP-9, BMP-7, and TGF- $\beta$ ligands may not be important endogenous regulators of hepcidin. Future research will be needed to definitively determine which TGF- $\beta$ /BMP superfamily ligands function as endogenous regulators of hepcidin expression and systemic iron balance, as well as determine the source of these ligands in vivo.

Anemia of chronic disease is associated with hypoferremia and reticuloendothelial cell iron sequestration. Inflammatory cytokines are potent inducers of hepcidin expression, and hepcidin excess is believed to have a key role in the pathogenesis of anemia in these patients (1-6). Presumably, inhibitors of hepcidin expression would allow for increased availability of iron from the diet and increased mobilization of iron from the spleen, thereby improving red blood cell production and ameliorating anemia. Here we provide in vivo evidence showing that soluble HJV.Fc inhibits BMP signaling in the liver, inhibits hepcidin expression, increases ferroportin protein expression, decreases splenic iron stores, and increase serum iron levels. This suggests that HJV.Fc is a potential new treatment for anemia associated with hepcidin excess.

We hypothesize that inhibition of hepatic BMP signaling is the predominant mechanism by which HJV.Fc inhibits hepcidin expression and regulates systemic iron balance in vivo. Indeed, inhibition of endogenous BMP signaling in HepG2 cells using BMP siRNAs had a similar effect on decreasing hepcidin expression as treatment with HJV.Fc. Furthermore, loss of TGF- $\beta$ /BMP superfamily signaling in the liver is sufficient to reduce hepcidin expression and generate iron overload, as demonstrated in mice with a liver-specific conditional knockout of Smad4 (23). However, hemojuvelin has been shown to bind to the receptor neogenin, a member of the deleted in colon cancer (DCC) receptor group, which has been reported to have a role in diverse functions including cell survival, axonal guidance, and cellular iron uptake (41). Whether this or other mechanisms contribute to HJV.Fc inhibition of hepcidin and alterations of systemic iron balance remains to be determined. Treatment with HJV.Fc in our study did not appear to have any other adverse effects on mice. Indeed, regulation of hepcidin expression and iron metabolism appears to be the principal role for TGF- $\beta$ /BMP superfamily signaling in the adult liver in vivo, since iron overload was the predominant phenotype of liver-specific conditional Smad4 knockout mice (23).

Recent studies suggest that inflammatory mediators such as IL-6 regulate hepcidin expression through STAT3 (42-44). Although the relationship between the IL-6/STAT3 and BMP/SMAD signaling pathways in the regulation of hepcidin expression is still poorly understood, mice with a liver-specific conditional knockout of Smad4 demonstrate attenuated hepcidin induction in response to IL-6 (23). This suggests that BMP/TGF- $\beta$ superfamily signaling is also necessary for hepcidin excess in inflammatory states and that inhibition of BMP signaling with HJV.Fc might attenuate hepcidin excess induced by inflammatory states. Here we show that HJV.Fc inhibits hepcidin induction in response to 
the inflammatory cytokine IL-6, consistent with prior reports for recombinant soluble hemojuvelin (33). Taken together, our results suggest that HJV.Fc or other inhibitors of BMP signaling may prove to be viable treatments for anemia of chronic disease caused by inflammation.

\section{Methods}

cDNA subcloning. To generate cDNA encoding HJV.Fc, an upstream fragment of human hemojuvelin (containing a preprotrypsin leader sequence and FLAG tag) was digested SpeI/BstEII from the plasmid FLAG-HJV (22). A downstream fragment of human hemojuvelin that does not include the glycophosphatidylinositol domain was amplified by PCR from the plasmid FLAG-HJV using the primers 5 '-AGAAGGTGTATCAGGCTGAGGTGG-3' and $5^{\prime}$-CAGCTCGAGTGAGGGGAAGAGATGCAGCTTCTC-3' , followed by BstEII/XhoI digestion. Both fragments were ligated into the SpeI/XhoI sites of Signal pIg plus (R\&D Systems) in-frame with the Fc portion of human IgG. Sequences were verified by bidirectional sequencing at the DNA sequencing core facility of Massachusetts General Hospital.

Purification of HJV.Fc. HEK293 cells (catalog no. CRL-1573; ATCC) cultured in RPMI medium 1640 (Invitrogen) supplemented with L-glutamine (Invitrogen) and 10\% FBS (Atlanta Biologicals) were stably transfected with cDNA encoding HJV.Fc using Lipofectamine 2000 (Invitrogen) according to the manufacturer's instructions. Stably transfected cells were selected and cultured in $1 \mathrm{mg} / \mathrm{ml}$ geneticin (Mediatech Inc.). HJV.Fc was purified from the conditioned media of stably transfected cells by Bio Express Cell Culture Services.

Animals. All animal protocols were approved by the Institutional Animal Care and Use Committee at the Massachusetts General Hospital or Children's Hospital Boston. Six- to 8-week-old 129S6/SvEvTac mice (Taconic) were housed in the Massachusetts General Hospital or Children's Hospital Boston rodent facilities and fed on either the Prolab 5P75 Isopro RMH 3000 or Prolab RMH 3000 diet (LabDiet), each with 380 parts per million iron.

BMP injection. Mice were anesthetized with avertin (Sigma-Aldrich) and given a single retroorbital injection of either $1 \mathrm{mg} / \mathrm{kg}$ of BMP-2 (kindly provided by Vicki Rosen, Harvard School of Dental Medicine, Boston, Massachusetts, USA) in $0.1 \%$ BSA in $1 \times$ PBS or an equal volume of vehicle alone. Dosage of BMP-2 was based on published data for BMP-7 showing that doses ranging from $0.25-1.0 \mathrm{mg} / \mathrm{kg}$ i.v. or i.p. are effective in inhibiting fibrosis and preserving renal function in several animal models of kidney injury (45-49). Four hours after injection the mice were sacrificed, and blood and livers were harvested for measurement of iron parameters and hepcidin expression.

HJV.Fc injection. Mice were injected with an i.p. dose of $25 \mathrm{mg} / \mathrm{kg}$ purified HJV.Fc or an equal volume of normal saline 3 times per week for 3 weeks. Twenty-four hours after the last injection, mice were sacrificed, and blood, livers, and spleen were harvested for measurement of iron parameters, phosphorylated Smad1, Smad5, and Smad8, and hepcidin expression.

Serum iron measurements. Blood was collected in BD Microtainer serum separator tubes (Fischer Scientific), and serum was isolated according to the manufacturer's instructions. Serum iron and unsaturated iron-binding capacity (UIBC) were measured by colorimetric assay using the Iron/UIBC kit (Thermo Electron Corp.). Total iron-binding capacity (TIBC) was calculated as the sum of serum iron and UIBC measurements, and transferrin saturation percentage was calculated as serum iron / TIBC $\times 100$.

Tissue iron measurement. Immediately after harvest, livers and spleen were sectioned and weighed. Quantitative measurement of nonheme iron was performed according to the method of Torrence and Bothwell (50). Results are reported as micrograms iron/gram wet weight tissue.

Luciferase assay. Hepcidin promoter luciferase assays in hepatomaderived Hep3B cells were carried out using the Dual-Luciferase Reporter
Assay System (Promega) as previously described (22) with the following modifications: for BMP/TGF- $\beta$ stimulation assays, cells transfected with the hepcidin promoter luciferase reporter and control Renilla luciferase vector ( $\mathrm{pRL}$-TK) were serum starved in $\alpha$-MEM with L-glutamine (Invitrogen) supplemented with 1\% FBS for 6 hours, followed by stimulation with $50 \mathrm{ng} / \mathrm{ml}$ BMP ligands, $30 \mathrm{ng} / \mathrm{ml}$ activin A, or $5 \mathrm{ng} / \mathrm{ml} \mathrm{TGF}-\beta$ ligands (R\&D Systems) for 16 hours. Relative concentrations of BMP/ TGF- $\beta$ superfamily ligands were similar to those previously used by others to compare superfamily ligand responses (23, 30-31). For HJV.Fc inhibition assays, cells transfected with the hepcidin promoter luciferase reporter and PRL-TK were serum starved as above and incubated with $25 \mathrm{ng} / \mathrm{ml}$ BMP-2, BMP-4, BMP-6, or BMP-7 ligands, $50 \mathrm{ng} / \mathrm{ml}$ BMP-5, or $5 \mathrm{ng} / \mathrm{ml}$ BMP-9 either alone or with $0.5-25.0 \mu \mathrm{g} / \mathrm{ml}$ of HJV.Fc for 16 hours. Relative concentrations of BMP ligands were chosen to elicit similar degrees of hepcidin promoter relative luciferase activity. Experiments using equal concentrations of ligands were also carried out and had similar results (data not shown).

RT-PCR. Total RNA was isolated from HepG2 or Hep3B cells and was analyzed for $B M P 2, B M P 4, B M P 5, B M P 6$, and $B M P 9$ expression as previously described (29) using the primers BMP-2 forward and reverse, BMP-4 forward and reverse, BMP- 6 forward and reverse, and BMP-9 forward and reverse (see Supplemental Table 1 for primer sequences; supplemental data available online with this article; doi:10.1172/31342DS1).

Quantitative real-time RT-PCR. Hep3B or HepG2 cells were serum starved for 6 hours in $\alpha$-MEM supplemented with 1\% FBS and treated for 16 hours with varying amounts of BMP/TGF- $\beta$ superfamily ligands or $100 \mathrm{ng} / \mathrm{ml} \mathrm{IL-6}$ in the absence or presence of $25 \mu \mathrm{g} / \mathrm{ml}$ purified HJV. Fc. For BMP siRNA experiments, HepG2 cells were plated in 24-well plates and transfected with $200 \mathrm{ng}$ pcDNA3 (Invitrogen) and $40 \mathrm{nM}$ BMP-2, BMP-4, BMP-6, BMP-7, or control scramble siRNA (Ambion; see Supplemental Table 2 for siRNA sequences) in $\alpha$-MEM using Lipofectamine 2000 (Invitrogen) according to the manufacturer's instructions. Cells were serum starved overnight in $\alpha$-MEM supplemented with $0.1 \%$ BSA. Total RNA was isolated from treated cells, and real-time quantitation of hepcidin relative to $\beta$-actin mRNA transcripts was performed using 2-step quantitative real-time RT-PCR as previously described (22). For BMP siRNA experiments, real-time quantitation of BMP2, BMP4, and $B M P 6$ relative to $\beta$-actin mRNA transcripts was also performed as described above using the primers qBMP- 2 forward, qBMP- 2 reverse, qBMP-4 forward, qBMP- 4 reverse, qBMP- 6 forward, and qBMP- 6 reverse (see Supplemental Table 3 for primer sequences). For mouse livers, total RNA was isolated using the Illustra RNAspin Mini Kit (GE Healthcare) according to the manufacturer's instructions. Real-time quantification of hepcidin (Hamp1) relative to Gapdh mRNA transcripts was performed as described above using primers Hamp1 forward (6) Hamp1 reverse (6), Gapdh forward, and Gapdh reverse (see Supplemental Table 3 for primer sequences).

Western blot. Western blot of purified HJV.Fc using anti-hemojuvelin antibody (22) and anti-Fc antibody (Jackson ImmunoResearch Laboratories) was performed as previously described (22). Western blot of liver lysates for phosphorylated Smad1, Smad5, and Smad8 expression relative to total Smad1 and $\beta$-actin expression was performed as previously described (22). Chemiluminescence was quantitated using IPLab Spectrum software version 3.9.5 r2 (Scanalytics). For ferroportin assays, spleen membrane preparations were prepared as previously described (51). Protein concentrations were determined by BCA assay (Pierce). After solubilization in $1 \times$ Laemmli buffer for 30 minutes at room temperature, $35 \mu \mathrm{g}$ of protein per sample were separated by SDS-PAGE using pre-cast NuPAGE Novex $4-12 \%$ Bis-Tris gels (Invitrogen) and transferred onto PDVF membranes. Western blot was performed using anti-ferroportin 
antibody (kindly donated by Francois Canonne-Hergaux) as previously described (52). Blots were stripped and reprobed for $\beta$-actin expression as a loading control as previously described (22).

Statistics. A 2-tailed Student's $t$ test with $P<0.05$ was used to determine statistical significance.

\section{Acknowledgments}

J.L. Babitt was supported in part by NIH grant K08 DK-075846. F.W. Huang was supported in part by NIH grant T32 HL07623. N.C. Andrews was supported in part by NIH grant RO1 DK-053813. H.Y. Lin was supported in part by NIH grants RO1
DK-69533 and RO1 DK-71837 and a grant from the Roche Foundation for Anemia Research.

Received for publication December 26, 2006, and accepted in revised form April 10, 2007.

Address correspondence to: Jodie L. Babitt, Program in Membrane Biology, Division of Nephrology, Massachusetts General Hospital, 185 Cambridge St., CPZN-8206, Boston, Massachusetts 02114, USA. Phone: (617) 643-3181; Fax: (617) 643-3182; E-mail: jbabitt@partners.org.
1. Weiss, G., and Goodnough, L.T. 2005. Anemia of chronic disease. N. Engl. J. Med. 352:1011-1023.

2. Pigeon, C., et al. 2001. A new mouse liver-specific gene, encoding a protein homologous to human antimicrobial peptide hepcidin, is overexpressed during iron overload. J. Biol. Chem. 276:7811-7819.

3. Nicolas, G., et al. 2002. The gene encoding the iron regulatory peptide hepcidin is regulated by anemia, hypoxia, and inflammation. J. Clin. Invest. 110:1037-1044. doi:10.1172/JCI200215686.

4. Nemeth, E., et al. 2004. IL-6 mediates hypoferremia of inflammation by inducing the synthesis of the iron regulatory hormone hepcidin. J. Clin. Invest. 113:1271-1276. doi:10.1172/JCI200420945.

5. Nemeth, E., et al. 2003. Hepcidin, a putative mediator of anemia of inflammation, is a type II acutephase protein. Blood. 101:2461-2463.

6. Lee, P., Peng, H., Gelbart, T., Wang, L., and Beutler, E. 2005. Regulation of hepcidin transcription by interleukin-1 and interleukin-6. Proc. Natl. Acad. Sci.U.S. A. 102:1906-1910.

7. Hentze, M.W., Muckenthaler, M.U., and Andrews, N.C. 2004. Balancing acts: molecular control of mammalian iron metabolism. Cell. 117:285-297.

8. Krause, A., et al. 2000. LEAP-1, a novel highly disulfide-bonded human peptide, exhibits antimicrobial activity. FEBS Lett. 480:147-150.

9. Park, C.H., Valore, E.V., Waring, A.J., and Ganz, T. 2001. Hepcidin, a urinary antimicrobial peptide synthesized in the liver. J. Biol. Chem. 276:7806-7810.

10. Nemeth, E., et al. 2004. Hepcidin regulates cellular iron efflux by binding to ferroportin and inducing its internalization. Science. 306:2090-2093.

11. Pietrangelo, A. 2006. Hereditary hemochromatosis. Biochim. Biophys. Acta. 1763:700-710.

12. Roetto, A., et al. 2003. Mutant antimicrobial peptide hepcidin is associated with severe juvenile hemochromatosis. Nat. Genet. 33:21-22.

13. Papanikolaou, G., et al. 2004. Mutations in HFE2 cause iron overload in chromosome 1q-linked juvenile hemochromatosis. Nat. Genet. 36:77-82.

14. Huang, F.W., Pinkus, J.L., Pinkus, G.S., Fleming, M.D., and Andrews, N.C. 2005. A mouse model of juvenile hemochromatosis. J. Clin. Invest. 115:2187-2191. doi:10.1172/JCI25049.

15. Niederkofler, V., Salie, R., and Arber, S. 2005. Hemojuvelin is essential for dietary iron sensing, and its mutation leads to severe iron overload. J. Clin. Invest. 115:2180-2186. doi:10.1172/JCI25683.

16. Ahmad, K.A., et al. 2002. Decreased liver hepcidin expression in the Hfe knockout mouse. Blood Cells Mol. Dis. 29:361-366.

17. Bridle, K.R., et al. 2003. Disrupted hepcidin regulation in HFE-associated haemochromatosis and the liver as a regulator of body iron homoeostasis. Lancet. 361:669-673.

18. Muckenthaler, M., et al. 2003. Regulatory defects in liver and intestine implicate abnormal hepcidin and Cybrd 1 expression in mouse hemochromatosis. Nat. Genet. 34:102-107.

19. Nicolas, G., et al. 2003. Constitutive hepcidin expression prevents iron overload in a mouse model of hemochromatosis. Nat. Genet. 34:97-101.

20. Kawabata, H., et al. 2005. Expression of hepcidin is down-regulated in TfR2 mutant mice manifesting a phenotype of hereditary hemochromatosis. Blood. 105:376-381.

21. Nemeth, E., Roetto, A., Garozzo, G., Ganz, T., and Camaschella, C. 2005. Hepcidin is decreased in TFR2 hemochromatosis. Blood. 105:1803-1806.

22. Babitt, J.L., et al. 2006. Bone morphogenetic protein signaling by hemojuvelin regulates hepcidin expression. Nat. Genet. 38:531-539.

23. Wang, R.H., et al. 2005. A role of SMAD4 in iron metabolism through the positive regulation of hepcidin expression. Cell Metab. 2:399-409.

24. Truksa, J., Peng, H., Lee, P., and Beutler, E. 2006. Bone morphogenetic proteins 2, 4, and 9 stimulate murine hepcidin 1 expression independently of Hfe, transferrin receptor 2 (Tfr2), and IL-6. Proc. Natl. Acad. Sci. U. S. A. 103:10289-10293.

25. Shi, Y., and Massague, J. 2003. Mechanisms of TGFbeta signaling from cell membrane to the nucleus. Cell. 113:685-700.

26. Monnier, P.P., et al. 2002. RGM is a repulsive guidance molecule for retinal axons. Nature. 419:392-395.

27. Samad, T.A., et al. 2004. DRAGON: a member of the repulsive guidance molecule-related family of neuronal- and muscle-expressed membrane proteins is regulated by DRG11 and has neuronal adhesive properties. J. Neurosci. 24:2027-2036.

28. Samad, T.A., et al. 2005. DRAGON, a bone morphogenetic protein co-receptor. J. Biol. Chem. 280:14122-14129.

29. Babitt, J.L., et al. 2005. Repulsive guidance molecule (RGMa), a DRAGON homologue, is a bone morphogenetic protein co-receptor. J. Biol. Chem. 280:29820-29827.

30. Korchynskyi, O., and ten Dijke, P. 2002. Identification and functional characterization of distinct critically important bone morphogenetic proteinspecific response elements in the Id 1 promoter. J. Biol. Chem. 277:4883-4891.

31. Dennler, S., et al. 1998. Direct binding of Smad3 and Smad4 to critical TGF beta-inducible elements in the promoter of human plasminogen activator inhibitor-type 1 gene. EMBO J. 17:3091-3100.

32. Moreland, L.W., et al. 1997. Treatment of rheumatoid arthritis with a recombinant human tumor necrosis factor receptor ( $\mathrm{p} 75)$-Fc fusion protein. N. Engl. J. Med. 337:141-147.

33. Lin, L., Goldberg, Y.P., and Ganz, T. 2005. Competitive regulation of hepcidin mRNA by soluble and cell-associated hemojuvelin. Blood. 106:2884-2889.

34. Roy, C.N., et al. 2004. An Hfe-dependent pathway mediates hyposideremia in response to lipopolysaccharide-induced inflammation in mice. Nat. Genet. 36:481-485

35. Wyeth Pharmaceuticals Inc. 2004. Summary of safety and effectiveness data for INFUSE bone graft. http://www.fda.gov/cdrh/PDF4/p000054b.pdf.

36. De Bleser, P.J., Niki, T., Rogiers, V., and Geerts, A. 1997. Transforming growth factor-beta gene expression in normal and fibrotic rat liver. J. Hepatol.

\section{6:886-893.}

37. Kingsley, D.M. 1994. What do BMPs do in mammals? Clues from the mouse short-ear mutation. Trends Genet. 10:16-21.

38. Knittel, T., Fellmer, P., Muller, L., and Ramadori, G. 1997. Bone morphogenetic protein-6 is expressed in nonparenchymal liver cells and upregulated by transforming growth factor-beta 1. Exp. Cell Res. 232:263-269.

39. Su, A.I., et al. 2002. Large-scale analysis of the human and mouse transcriptomes. Proc. Natl. Acad. Sci. U. S. A. 99:4465-4470.

40. Miller, A.F., Harvey, S.A., Thies, R.S., and Olson, M.S. 2000. Bone morphogenetic protein-9. An autocrine/paracrine cytokine in the liver. J. Biol. Chem. 275:17937-17945.

41. Zhang, A.S., West, A.P., Jr. Wyman, A.E., Bjorkman, P.J., and Enns, C.A. 2005. Interaction of hemojuvelin with neogenin results in iron accumulation in human embryonic kidney 293 cells. J. Biol. Chem. 280:33885-33894.

42. Wrighting, D.M., and Andrews, N.C. 2006. Interleukin-6 induces hepcidin expression through STAT3. Blood. 108:3204-3209.

43. Verga Falzacappa, M.V., et al. 2007. STAT3 mediates hepatic hepcidin expression and its inflammatory stimulation. Blood. 109:353-358.

44. Pietrangelo, A., et al. 2007. STAT3 is required for IL-6-gp130-dependent activation of hepcidin in vivo. Gastroenterology. 132:294-300.

45. Bosukonda, D., Shih, M.S., Sampath, K.T., and Vukicevic, S. 2000. Characterization of receptors for osteogenic protein-1/bone morphogenetic protein-7 (OP-1/BMP-7) in rat kidneys. Kidney Int. 58:1902-1911.

46. Hruska, K.A., et al. 2000. Osteogenic protein-1 prevents renal fibrogenesis associated with ureteral obstruction. Am. J. Physiol. Renal Physiol. 279:F130-F143.

47. Morrissey, J., et al. 2002. Bone morphogenetic protein-7 improves renal fibrosis and accelerates the return of renal function. J. Am. Soc. Nephrol. 13(Suppl. 1):S14-S21.

48. Vukicevic, S., et al. 1998. Osteogenic protein-1 (bone morphogenetic protein-7) reduces severity of injury after ischemic acute renal failure in rat. J. Clin. Invest. 102:202-214.

49. Zeisberg, M., et al. 2003. Bone morphogenic protein-7 inhibits progression of chronic renal fibrosis associated with two genetic mouse models. Am. J. Physiol. Renal Physiol. 285:F1060-F1067.

50. Torrance, J.D., and Bothwell, T.H. 1980. Tissue iron stores. Methods Hematol. 1:90-115.

51. Canonne-Hergaux, F., Gruenheid, S., Ponka, P., and Gros, P. 1999. Cellular and subcellular localization of the Nramp2 iron transporter in the intestinal brush border and regulation by dietary iron. Blood. 93:4406-4417.

52. Canonne-Hergaux, F., Donovan, A., Delaby, C., Hui-jun, W., and Gros, P. 2005. Comparative studies of duodenal and macrophage ferroportin proteins. Am. J. Physiol. Gastrointest. Liver Physiol. 290:156-163. 